\title{
Extractions et caractérisations physico-chimiques des substances humiques naturelles des sols et du compost
}

\author{
Moursalou KORIKO ${ }^{1 *}$, Sanonka TCHEGUENI ${ }^{1}$, Komi Edem KOLEDZI ${ }^{1}$, \\ Dihéénane D. BAFAI ${ }^{1}$, Essey ZONVIDEY ${ }^{1}$, Gado TCHANGBEDJI ${ }^{1}$, \\ Ani Koffi KILI ${ }^{1}$ et Mohamed HAFIDI ${ }^{2}$ \\ ${ }^{1}$ Laboratoire de Gestion, Traitement et Valorisation des Déchets (G.T.V.D.), Faculté des Sciences, \\ B.P. 1515, Université de Lomé, Togo. \\ ${ }^{2}$ Laboratoire d'Ecologie, Sols et Environnement, Faculté des Sciences Semlalia, Département de Biologie, \\ B.P. 2390, Marrakech, Maroc. \\ *Auteur correspondant, E-mail: moursalou7@hotmail.com
}

\section{RESUME}

Les substances macromoléculaires naturelles qui constituent la matière organique (MO) des sols sont formées essentiellement au cours des processus de dégradation des matières végétales et animales par les microorganismes. Elles jouent un rôle dans la fertilisation, le transport, l'immobilisation et la dégradation des espèces chimiques organiques ou minérales telles que les pesticides, les composés aromatiques polycycliques ou les métaux lourds dans les sols et les eaux. Elles doivent ces rôles à leurs caractéristiques chimiques et structurales. D'un milieu à un autre, leurs structures et leurs caractéristiques diffèrent en fonction de conditions physico-chimiques telles que le $\mathrm{pH}$, le taux d'humidité, la température, la composition chimique etc. Dans le cadre de l'étude de la dissolution des phosphates naturels de Hahotoé-Kpogamé (Togo) par les acides organiques naturels, nous avons réalisé l'extraction des substances humiques d'un sol et d'un compost à l'aide d'une solution alcaline de $\mathrm{NaOH}(0,1 \mathrm{~N})$ après un prétraitement aqueux ou acide. Les échantillons extraits ont été purifiés par dialyse. La caractérisation de ces substances par des méthodes chimiques (dosages élémentaires et du Carbone Organique Total (COT)) et physiques (Spectroscopies Infrarouge et UV-Visible, Microscopie Electronique à Balayage (MEB)) a été effectuée. Les méthodes analytiques utilisées ont permis d'identifier les groupements fonctionnels qui confirment le caractère humique des échantillons extraits.

(C) 2013 International Formulae Group. All rights reserved.

Mots clés : Substances humiques, extraction, caractérisation, infrarouge, UV-Visible, Microscopie Electronique à Balayage (MEB).

\section{INTRODUCTION}

Les substances macromoléculaires naturelles qui constituent la matière organique des sols sont formées essentiellement au cours des processus de dégradation des matières végétales et animales par les microorganismes. Ces substances représentent une fraction importante du carbone organique dissout contenu dans les eaux interstitielles des sols $(60-70 \%)$ et dans les eaux lacustres, fluviales et marines (30-50\%) (Boris, 2004 ; Peña-Médez et al., 2005). Elles sont, sur le plan physico-chimique, les constituants du sol les moins connus malgré les nombreux 
travaux qui leur ont été consacrés (Calvet, 2003a, 2003b). Cette fraction de la matière organique est un mélange hétérogène de biopolymères de masses moléculaires comprises entre 1000 et 300000 daltons (Wolf et al., 2003). Outre leur rôle dans la fertilisation, elles jouent un rôle considérable dans le transport, l'immobilisation et la dégradation des espèces chimiques organiques ou minérales telles que les pesticides, les composés aromatiques polycycliques ou les métaux lourds dans les sols et les eaux. Elles doivent ce rôle à leurs caractéristiques chimiques et structurales. D'un milieu à un autre, leurs structures et leurs caractéristiques diffèrent en fonction de conditions physicochimiques telles que le $\mathrm{pH}$, le taux d'humidité, la température, la composition chimique etc. Les propriétés de la matière organique du sol et plus particulièrement des substances humiques, ne peuvent être bien étudiées qu'à l'état libre, donc débarrassées de ses composants inorganiques. C'est dans le cadre de l'étude de la dissolution des phosphates naturels de Hahotoé-Kpogamé (Togo) par les substances humiques naturelles que nous avons effectué ce travail qui constitue une première étape déterminante dans l'étude des propriétés acide-base et de complexation de celles-ci.

\section{MATERIEL ET METHODES}

\section{Les réactifs chimiques}

Les produits utilisés au cours de nos travaux sont des solutions et des produits solides d'origine commerciale. Les caractéristiques de ces produits sont décrites dans le Tableau 1.

\section{Extraction et purification des substances humiques}

\section{Extraction des substances humiques}

L'extraction des substances humiques est réalisée à l'aide d'une solution alcaline de $\mathrm{NaOH}(0,1 \mathrm{~N})$ après un prétraitement aqueux ou acide. La technique d'extraction que nous avons mise au point est schématisée sur la Figure 1. Pour l'extraction des substances humiques $\mathrm{du}$ compost, au cour $\mathrm{du}$ prétraitement, des échantillons (15 g) ont été délipidés par traitement de mélange de solvants chloroforme-méthanol dans un rapport $2: 1$; les résidus des échantillons sont évaporés sous azote pour éliminer le reste du solvant (Lichtfouse et al., 1998). Les échantillons ont ensuite été traités à de l'eau distillée ( 3 fois) pour éliminer les substances solubles non humiques (sucres, protéines) susceptibles d'interférer avec les substances humiques (Amir et al., 2003).

\section{Purification des produits}

Les substances humiques extraites, avant d'être analysées doivent être purifiées par dialyse. Cette technique permet de séparer les molécules selon leur poids moléculaire grâce à l'utilisation de membranes semiperméables dont les pores permettent aux particules de petite taille (ions, solvants) de diffuser à travers la membrane. Les molécules de taille plus importante sont conservées à l'intérieur de la membrane d'ester cellulose Da (Spectra/Por CE®) de porosité 1000. Enfin, les échantillons ont été lyophilisés pour éliminer l'eau.

\section{Les méthodes de caractérisation utilisées Dosage du Carbone Organique Total (COT)}

La mesure du Carbone Total Organique (COT) permet de déterminer la teneur en carbone. Ces mesures ont été réalisées à l'aide d'un analyseur SHIMADZU TOC 5050A.

Analyse spectroscopique Infrarouge (IR)

Les spectres infrarouges à transformer de Fourier de nos échantillons ont été enregistrés à l'aide d'un spectromètre PERKIN ELMER FT-IR, SPECTROMETER PARAGON 1000 dans le domaine de 4000 à $400 \mathrm{~cm}^{-1}$, en utilisant des pastilles de $\mathrm{KBr}(1$ $\mathrm{mg}$ d'échantillon d'acide humique pour $9 \mathrm{mg}$ de $\mathrm{KBr}$ solide). 


\section{Analyse spectroscopique UV-Visible}

Le rapport de Welt (ratio $\mathrm{E}_{4} / \mathrm{E}_{6}$ ) a été utilisé dans ce travail pour caractériser les substances humiques. Il est déterminé à partir de la mesure des absorbances à $472 \mathrm{~nm}\left(\mathrm{E}_{4}\right)$ et $665 \mathrm{~nm}\left(\mathrm{E}_{6}\right)$ des solutions humiques diluées. Nous avons enregistré les spectres d'absorption UV-visible de nos échantillons à l'aide d'un spectrophotomètre CARY 3 à double faisceau parallèle couplé à un ordinateur pour la mémorisation et le retraitement des spectres. La précision de l'appareil est de $\pm 0,002$ de densité optique. Les échantillons de SH ont été dilués dans de l'eau distillée pour atteindre une concentration comprise entre 200 et $300 \mathrm{mg} . \mathrm{L}^{-1}$ et leur $\mathrm{pH}$ a été ajusté entre 6 et 7.

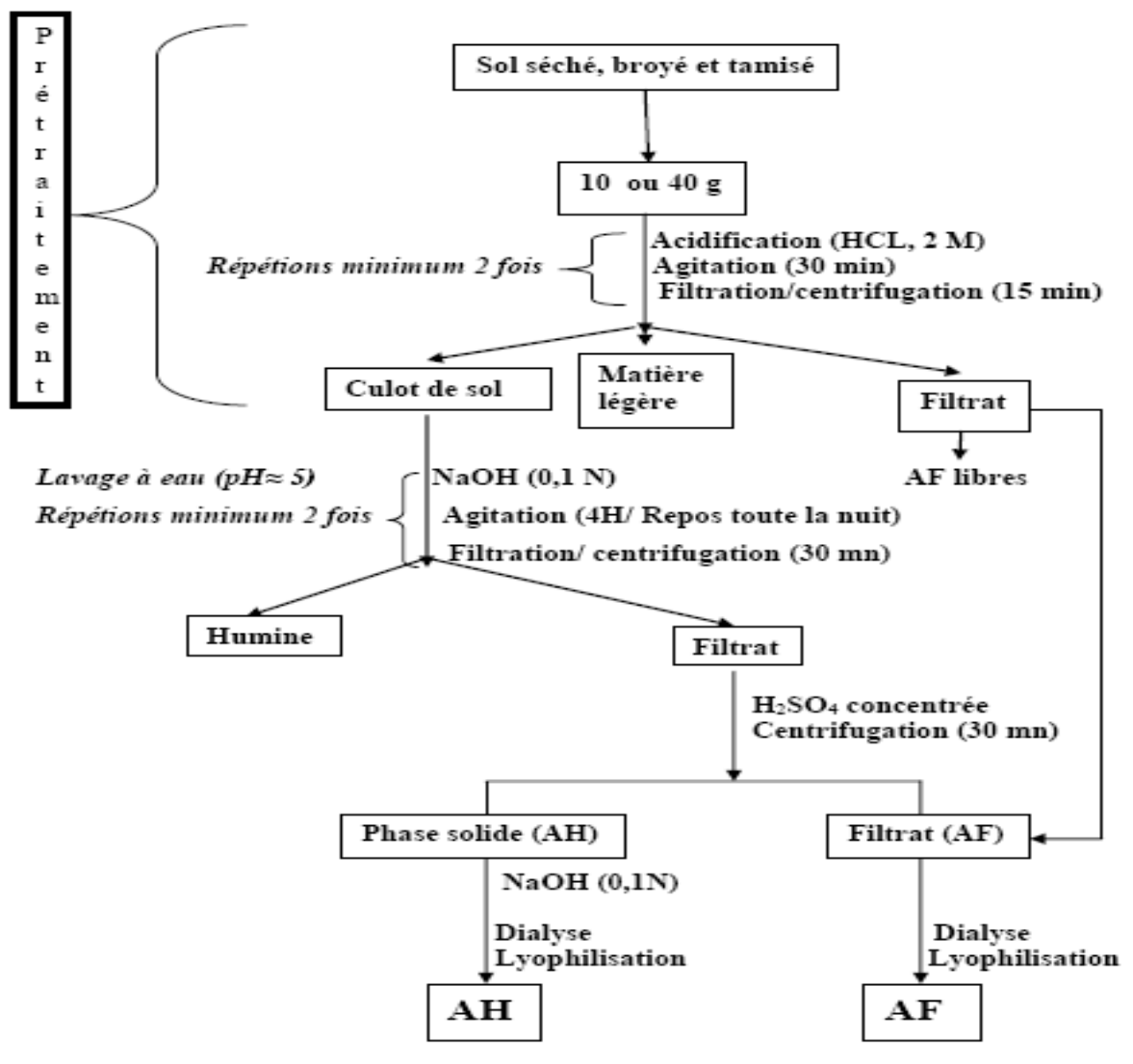

Figure 1: Schéma des différentes étapes d'extraction des substances humiques des sols.

Tableau 1: Caractéristiques des produits chimiques utilisés.

\begin{tabular}{|l|c|c|c|c|}
\hline \multicolumn{1}{|c|}{ Produits chimiques } & Origine & Pureté & Masse molaire (en g) & Densité \\
\hline Acide sulfurique $\left(\mathrm{H}_{2} \mathrm{SO}_{4}\right)$ & Prolabo-Normapur & $97 \%$ & 96,08 & 1,84 \\
\hline Acide chlorhydrique $(\mathrm{HCl})$ & Prolabo-Normapur & $65 \%$ & 63,01 & 1,40 \\
\hline Hydroxyde de sodium & Jeulin & $99,99 \%$ & 40,00 & - \\
\hline
\end{tabular}




\section{RESULTATS ET DISCUSSION}

Analyses macroscopique et microscopique des substances humiques

La présence des substances humiques dans un milieu est classiquement mise en évidence à travers leurs couleurs en solution (McCarthy et al., 1990).

Les solutions des différentes fractions des substances humiques extraites des sols et des composts présentent les colorations que nous avons reportées sur la Figure 2.

On peut ainsi constater sur les photos de cette figure que les acides fulviques ont une teinte très claire tendant vers le jaune similaire aux couleurs indiquées dans la littérature (Tableau 2), signe d'une faible teneur en carbone. Comme dans la littérature, les acides humiques ont une coloration noirâtre qui indique une forte concentration en carbone que celui du compost.

L'observation au Microscope Electronique à Balayage (M.E.B.) des différents échantillons des substances humiques purifiées par dialyse et lyophilisées, permet d'avoir une idée de leur structure tridimensionnelle. A faible grossissement (500 fois), les images se présentent comme des agrégats de molécules comme l'indiquent les clichés de la Figure 3. Cela est dû probablement aux traitements (lyophilisation et métallisation) qui donnent aux échantillons un aspect spongieux et léger après la sublimation des molécules d'eau. Des grossissements plus importants du même agrégat (2000 fois) laissent apparaître un enchevêtrement de filaments (cliché b) qui semble pouvoir se dérouler (cliché d) (Figure 4).

Comme on peut le constater sur la Figure 5, les acides humiques (cliché h) présentent une structure condensée et globulaire, et les acides fulviques, une structure moins dense (cliché j).

\section{Dosage du carbone organique total (COT)}

Il a été effectué sur les acides humiques très riches en matières organiques $\left(\mathrm{AHS}_{\mathrm{L}}\right.$ et $\mathrm{AHS}_{\mathrm{B}}$ ). Les résultats des teneurs en COT (différence entre la quantité du CT et celle du
CI) de ces échantillons dans les différentes solutions sont consignés dans le Tableau 3. Ils représentent la moyenne de trois essais effectués pour chaque type d'analyse. Cette valeur moyenne est obtenue avec un écart inférieur ou égal à $\pm 0,5 \mathrm{mg} \cdot \mathrm{L}^{-1}$. La limite de détection est de $50 \mathrm{ppb}$ et on peut mesurer jusqu'à 50 ppm en concentration de carbone.

Ces résultats révèlent la présence du carbone organique dans les échantillons humiques purifiés. Le dosage du COT a donné de meilleurs résultats avec les solutions de soude par rapport au tampon phosphaté.

\section{Analyse UV-Visible : rapport de Welt $\left(E_{4} /\right.$ $\left.\mathbf{E}_{6}\right)$ \\ Les résultats de l'analyse de nos} échantillons par la méthode spectroscopique UV- visible sont répertoriés sur le Tableau 4. Le ratio $\mathbf{E}_{\mathbf{4}} / \mathbf{E}_{\mathbf{6}}$ sert de paramètre d'interprétation de ces résultats. C'est ainsi que nous avons obtenu le ratio de certains de nos échantillons inférieur à 5. Il s'agit des acides humiques qui possèdent une longue chaîne carbonée et un poids moléculaire élevé.

Les ratios de nos acides fulviques sont supérieurs à 5 ; ce qui est en parfait accord avec la littérature qui attribue cet état au faible poids moléculaire de ces acides (Swift, 1996 ; Thomsen et al., 2002 ; Domeizel et al., 2003 ; Zbytniewski et al., 2005). Ils indiquent également une faible teneur en carbone, une forte teneur en oxygène, en groupement carboxylique et une acidité totale importante. Ces résultats confirment ceux de la détermination du COT.

\section{Analyse à l'Infra Rouge}

Les spectres infrarouges enregistrés entre 4000 et $400 \mathrm{~cm}^{-1}$ de nos échantillons son reportés à la Figures 6 . Les spectres obtenus présentent de grandes similitudes en ce qui concerne l'allure générale et les positions des pics principaux.

Les pics entre 3500-2800 $\mathrm{cm}^{-1}$ relativement larges, intenses et couvrant un domaine d'environ 500 à $600 \mathrm{~cm}^{-1}$ sont caractéristiques des substances humiques. Ils sont attribués principalement à l'absorbance 
des groupements $\mathrm{O}-\mathrm{H}$ des différentes fonctions, des groupements N-H (Miano et al., 1992 ; Niemeyer et al., 1992). Ceci est en parfait accord avec les modèles macromoléculaires proposés pour les matières humiques notamment par Schulten et al. (1992, 1993) qui ont supposé qu'une abondance de groupements hydroxyles et les possibilités d'enroulement favorisant l'établissement de liaisons hydrogènes intramoléculaires seraient à l'origine de la largeur du pic et de son intensité.

Le deuxième groupe de raies bien définies est situé dans la région de 1700$1500 \mathrm{~cm}^{-1}$. Selon certains auteurs, l'absorption des radiations IR dans cette région est principalement due aux vibrations de doubles liaisons $\mathrm{C}=\mathrm{C}$ dans les systèmes aromatiques conjugués avec les groupements carboxyliques ou carbonyles (De Nobili et al., 1990 ; Benny et al., 1996; Amir et al., 2006). Il a été signalé que les vibrations angulaires des liaisons $\mathrm{O}-\mathrm{H}$ dans la molécule d'eau absorbent dans une bande centrée à $1600 \mathrm{~cm}^{-1}$, ces vibrations pourraient donc contribuer à l'absorbance vers $1500 \mathrm{~cm}^{-1}$ pour nos échantillons qui ne sont pas du tout secs. Nous attribuons les pics moins intenses aux alentours de $1400 \mathrm{~cm}^{-1}$ aux structures aliphatiques. En effet, il est signalé dans la littérature que cette région correspondrait aux vibrations de valence des liaisons $\mathrm{C}-\mathrm{H}$ dans une structure aliphatique (Niemeyer et al., 1992).

Le dernier groupe de pics des spectres, légèrement décalé vers les basses fréquences $\left(1100\right.$ à $\left.1050 \quad \mathrm{~cm}^{-1}\right)$ serait dû aux déformations des liaisons $\mathrm{C}-\mathrm{O}$ et $\mathrm{C}-\mathrm{H}$ dans les structures ressemblant aux polysaccharides (Niemeyer et al., 1992). Enfin, l'existence des crêtes entre 520 et $470 \mathrm{~cm}^{-1}$ est due aux groupements aromatiques para-di-substitué.

\section{Détermination des constantes de dissociation acido-basiques}

Cette détermination des constantes est réalisée uniquement sur des acides humiques qui ont fait l'objet des études complexométriques. Elle nous a permis de faire une étude comparative des mécanismes de complexations des deux acides humiques.

Les constantes de dissociation $(\mathrm{Ka})$ sont des paramètres qui permettent d'évaluer les forces des acides. Le calcul de ces constantes est réalisé à partir du pH et de la concentration de la solution acide. Cependant, la difficulté de connaître avec précision les concentrations des solutions aussi complexes que celles des substances humiques conduit à utiliser la dérivé première $(\mathrm{pH} / \mathrm{V}(\mathrm{ml}))$ de la courbe potentiométrique en fonction du $\mathrm{pH}$, pour déterminer les $\mathrm{pKa}$ et les points de neutralisation des acides humiques (Tableau 5 et Figure 7).

Pour les deux acides humiques, les maxima correspondent aux points d'inflexion des courbes potentiométriques, c'est-à-dire aux points de neutralisation, 6,46 et 9,25 pour AHC et 4,89, 7,94 et 9,41 pour $\mathrm{AHS}_{\mathrm{B}}$.

La première acidité de $\mathrm{AHS}_{\mathrm{B}}$ serait vraisemblablement due aux fonctions carboxyliques aliphatiques (pKa de l'acide acétique est de 4,75) ou aromatiques (pKa de l'acide benzoïque est de 4,2, et de l'acide phtalique sont respectivement 3 et 5,3) alors que les acidités plus faibles peuvent être attribués surtout aux $\mathrm{OH}$ phénoliques ( $\mathrm{pKa} \mathrm{du}$ phénol est de 10,00; celui du pyrocatéchol (1,2-hydroxybenzène) est 9,5, celui du résorcinol (1,3-dihydroxybenzène) est de $9,15)$ et dans une moindre mesure, à des $\mathrm{OH}$ alcooliques ou à des groupes amino ( $\mathrm{pKa}$ de la n-butylamine est de 10,6). Les groupes carboxyliques et phénoliques et surtout leurs positions respectives au sein des macromolécules des $\mathrm{SH}$, jouent un rôle décisif dans la formation des complexes organométalliques. Les valeurs des $\mathrm{pKa}$ qui caractérisent le degré de dissociation des groupements fonctionnels des acides humiques à des $\mathrm{pH}$ déterminés, diffèrent en fonction de l'origine de ceux-ci et aussi de leur mode d'extraction (Amir et al., 2003). 


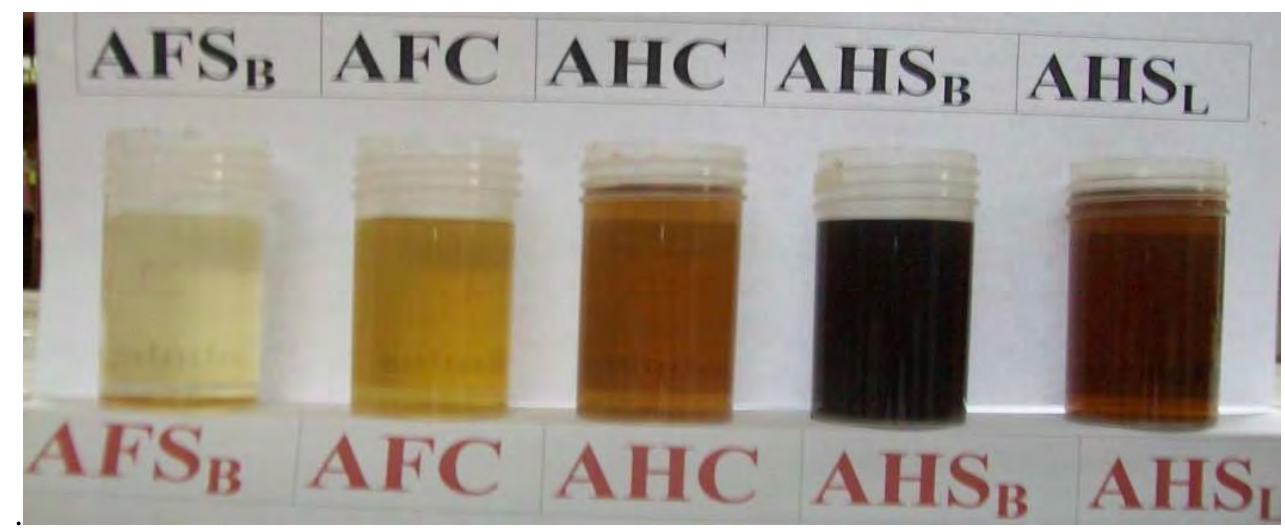

Figure 2: Couleurs des solutions des différentes substances humiques à des concentrations de 200 $\mathrm{mg} / \mathrm{L}$. Acides fulviques [AFC et AFS] ; acides humiques [AHC et $\left.\mathrm{AHS}_{\mathrm{L}}, \mathrm{AHS}_{\mathrm{B}}\right]$.
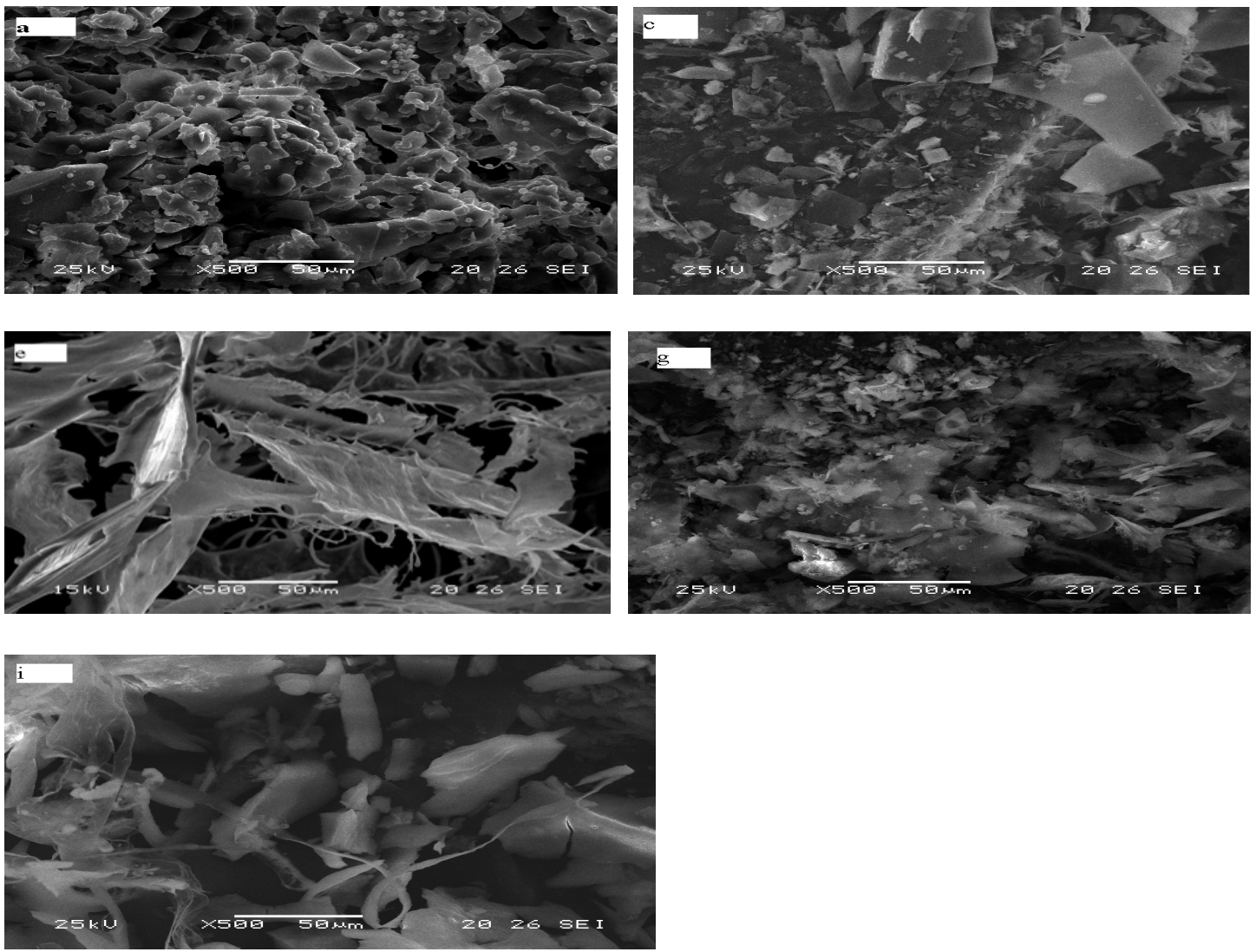

Figure 3 : Clichés de substances humiques observées en Microscopie Electronique à Balayage avec un grossissement de 500 fois. a) $\mathrm{AHC}$, c) $\mathrm{AH}_{\mathrm{L}}$, e) $\mathrm{AHS}_{\mathrm{B}}$, g) $\mathrm{AFC}$, i) $\mathrm{AFS}_{\mathrm{B}}$. 


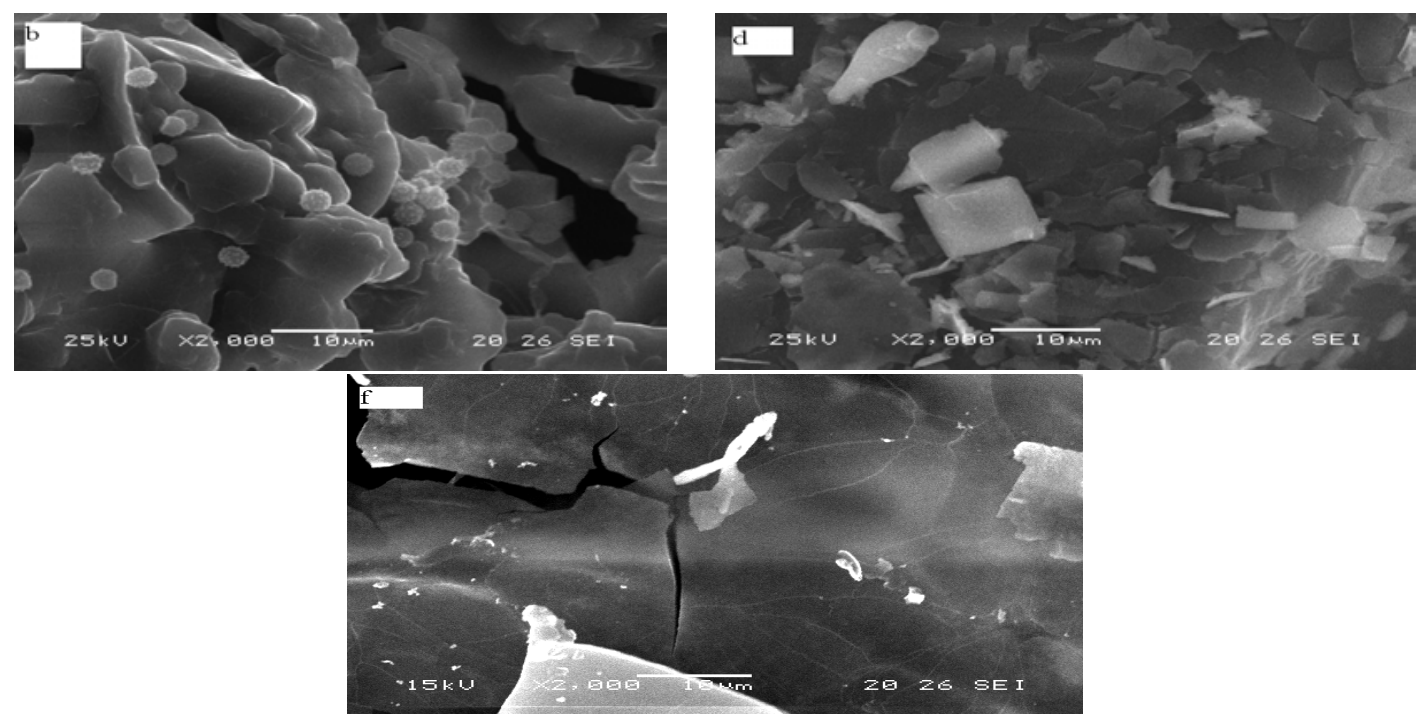

Figure 4 : Clichés de substances humiques observées en Microscopie Electronique à Balayage avec un grossissement de 2000 fois. b) $\mathrm{AHC}$, d) $\mathrm{AHS}_{\mathrm{L}}$, f) $\mathrm{AHS}_{\mathrm{B}}$.
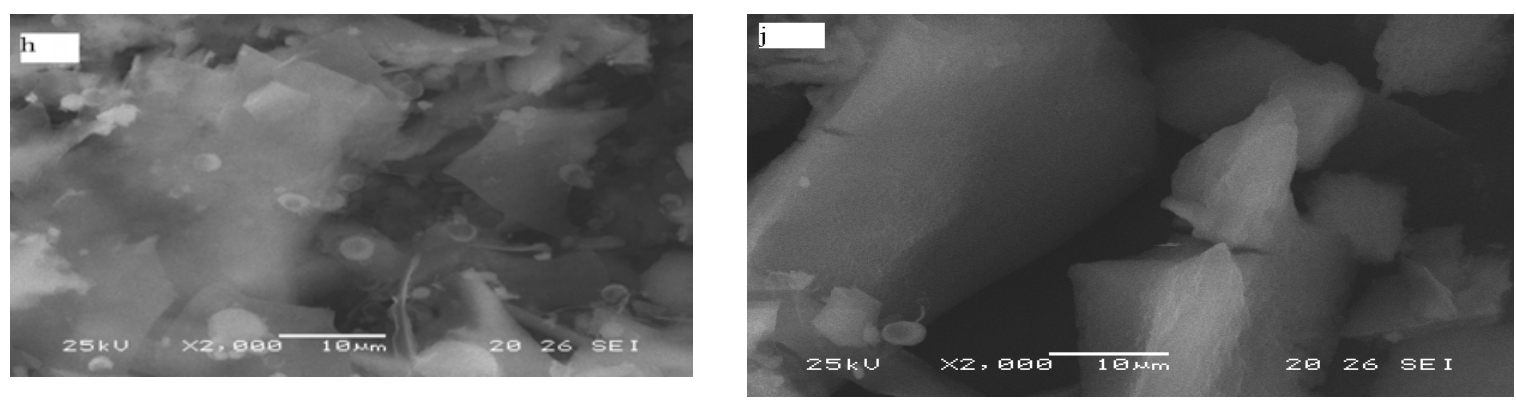

Figure 5: Clichés $\mathrm{AFC}$ (cliché h) et $\mathrm{AFS}_{\mathrm{B}}$ (cliché i) observées en Microscopie Electronique à Balayage avec un grossissement de 2000 fois.
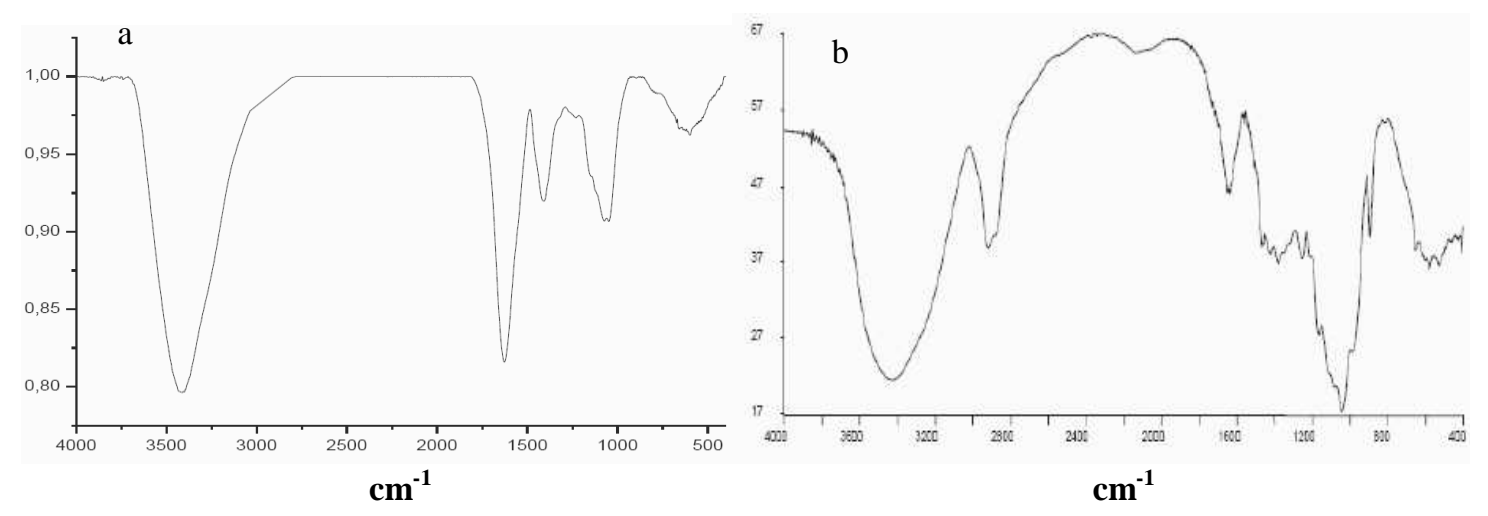

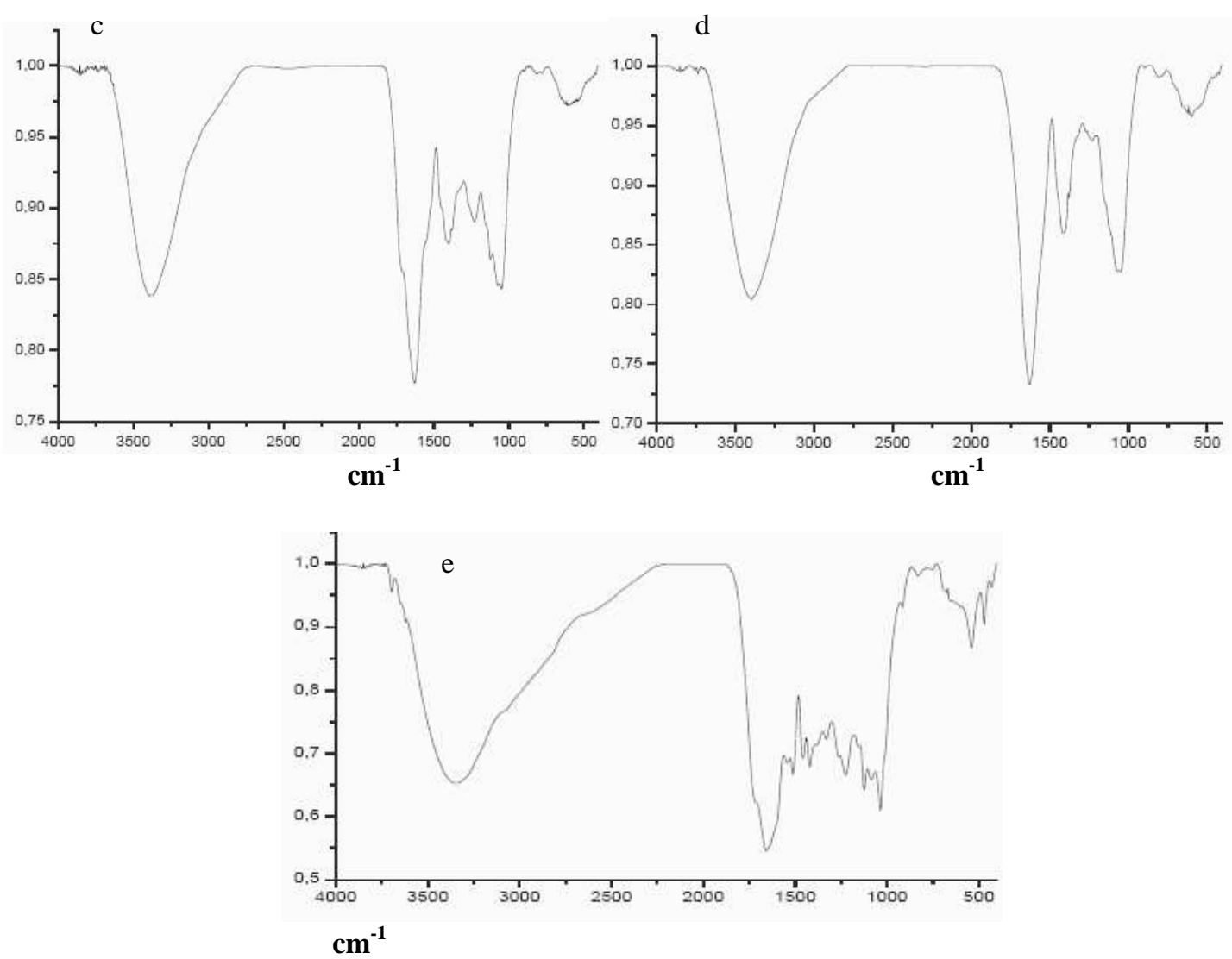

Figure 6: Les spectres infrarouges des échantillons analysés. (a) acide humique du sol de Badou ( $\mathrm{AHS}_{\mathrm{B}}$ ), (b) acide humique du sol de Lomé ( $\mathrm{AHS}_{\mathrm{L}}$ ), (c) acide humique du compost (AHC), (d) acide fulvique du sol de Badou (AFS $\mathrm{B}$ ), (e) de l'acide fulvique du compost (AFC).
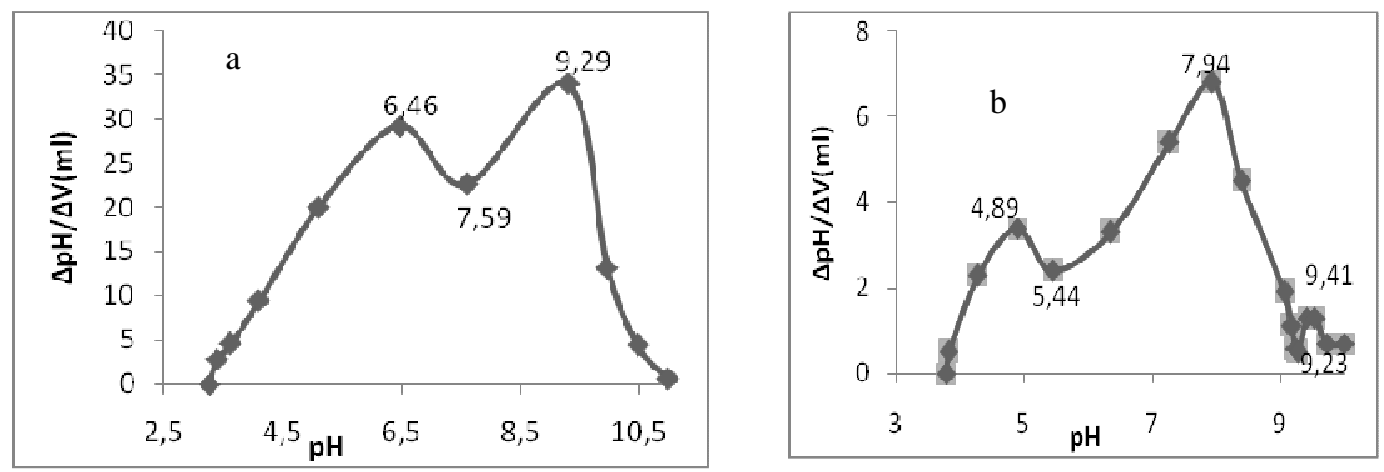

Figure 7: Courbes de neutralisation des acides humiques par la soude à 0,042 N. (a) acide humique du compost ; b) acide humique du sol. 
Tableau 2 : Evolution des couleurs des substances humiques (Stevenson et al., 1992).

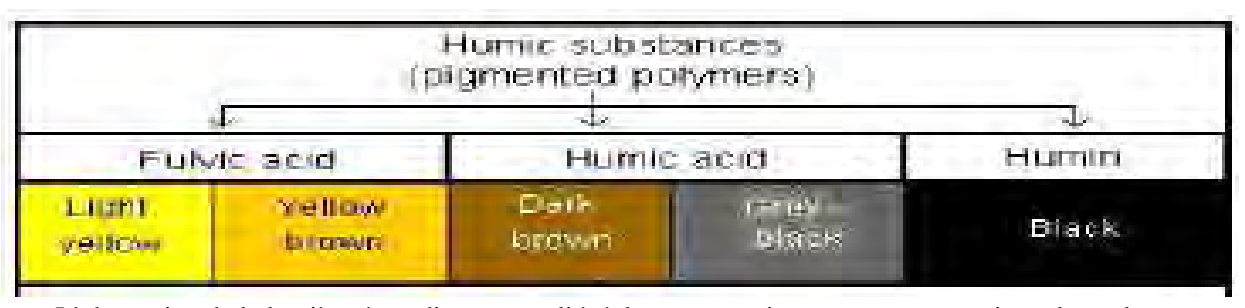

L'absorption de la lumière étant directement liée à la concentration en noyau aromatique, la couleur des substances humiques peut fournir des indications sur la taille des molécules.

Tableau 3: Teneurs du COT des acides humiques dans les différentes solutions.

\begin{tabular}{lcc}
\hline Carbone & \multicolumn{2}{c}{ Tampon phosphate $(\mathbf{p H}=\mathbf{6 , 5})+\mathbf{A H}$} \\
\hline $\mathrm{AH}$ & $\mathrm{AHS}_{\mathrm{L}}\left(50 \mathrm{mg} \cdot \mathrm{L}^{-1}\right)$ & $\mathrm{AHS}_{\mathrm{B}}\left(50 \mathrm{mg} \cdot \mathrm{L}^{-1}\right)$ \\
$\mathrm{CT}(\mathrm{mg} / \mathrm{l})$ & 13,63 & 17,02 \\
$\mathrm{CI}(\mathrm{mg} / \mathrm{l})$ & 0,235 & 0,588 \\
$\mathrm{COT}(\mathrm{mg} / \mathrm{l})$ & 13,39 & 16,43 \\
$\%$ COT & $26,78 \%$ & $32,86 \%$ \\
\hline \multicolumn{2}{c}{$\mathrm{AHS}_{\mathrm{L}}=$ acide humique du sol de Lomé $; \mathrm{AHS}_{\mathrm{B}}$ : acide humique du sol de Badou }
\end{tabular}

Tableau 4: Ratio $\mathrm{E}_{4} / \mathrm{E}_{6}$ des différentes substances humiques.

\begin{tabular}{cccccc}
\hline & $\mathbf{A H C}$ & $\mathbf{A H S}_{\mathbf{B}}$ & $\mathbf{A H S}_{\mathbf{L}}$ & $\mathbf{A F S}_{\mathbf{B}}$ & $\mathbf{A F C}$ \\
\hline $\mathrm{E}_{4} / \mathrm{E}_{6}$ & 4,895 & 4,185 & 4,965 & 6,165 & 9,420 \\
\hline AFC= acide fulvique du compost, $\mathrm{AHC}=$ acide humique du compost, \\
AHS $\mathrm{L}_{\mathrm{L}=\text { acide humique du sol de Lomé, }} \mathrm{AHS}_{\mathrm{B}}=$ acide humique du sol de Badou.
\end{tabular}

Tableau 5: Points de neutralisation et des valeurs de pKa des acides humiques.

\begin{tabular}{cccccc}
\hline & \multicolumn{3}{c}{ Points de neutralisation } & \multicolumn{2}{c}{ Valeurs de pKa } \\
\hline & $1^{\text {er }}$ point & $2^{\text {eme }}$ point & $3^{\text {eme }}$ point & $\mathrm{pKa}_{1}$ & $\mathrm{pKa}_{2}$ \\
$\mathrm{AHC}$ & 6,46 & 9,25 & ------- & 7,59 & -------- \\
$\mathrm{AHS}_{\mathrm{B}}$ & 4,89 & 7,94 & 9,41 & 5,44 & 9,23 \\
\hline Les minima correspondent & aux pKa des acides c'est-à-dire, $\mathrm{pKa}_{1}=7,59$ pour $\mathrm{AHC}$ et $\mathrm{pKa}_{1}=5,44$ et $\mathrm{pKa}_{2}=9,23$ pour $\mathrm{AHS}_{\mathrm{B}}$.
\end{tabular}

\section{Conclusion}

L'étude de la nature chimique et de la structure des molécules humiques constitue un premier axe fondamental pour comprendre les propriétés physiques, chimiques et agronomiques de celles-ci. Cette première phase de notre travail a porté sur l'extraction et la caractérisation des structures d'acides humiques et fulviques en se basant sur les différentes techniques: analyses macroscopiques, microscopiques et élémentaires et la spectroscopie Infrarouge à Transformer de Fourrier (FTIR). Toutes ces analyses se sont complétées et ont donné des résultats convergents.

L'analyse élémentaire et le rapport de Welt montrent que les structures des substances humiques contiennent de teneurs élevées en $\mathrm{C}$ et $\mathrm{O}$ puis de faibles teneurs en $\mathrm{N}, \mathrm{H}$ et $\mathrm{S}$. Toutes ces valeurs sont presque 
dans l'ordre des valeurs rencontrées dans la littérature.

Un faible rapport $\mathrm{E}_{4} / \mathrm{E}_{6}$ de Welt suggère la composition des acides fulviques en structures condensées et chez les acides humiques, il est lié à une diminution de leur teneur en oxygène ou une augmentation de leur degré de polycondensation. L'analyse par spectroscopie infrarouge confirme aussi une forte présence des structures aromatiques dans des substances humiques avec des chaînes alkyles latérales polyalcools ou polyacides. Les méthodes analytiques utilisées dans ce chapitre ont permis d'identifier les groupements fonctionnels qui confirment le caractère humique des échantillons extraits.

\section{REFERENCES}

Amir S, Hafidi M, Lemee L, Merlina G, Guiresse M, Pinelli, E, Revel JC, Bailly JR, Ambles. 2006. Structural characterization of humic acids, extracted from sewage sludge during composting, by thermochemolysis - gas chromatography-mass spectrometry. Process Biochemistry, 41: 410-422.

Amir S, Hafidi M, Bailly JR, Revel JC. 2003. Characterization of humic acids extracted from sewage sludge during composting and of their Sephadex gel fractions. Agronomie, 23: 269-275.

Benny C, Hatchery PG, Hagar Y, Chen. 1996. Chemical and biological characterization of organic matter during composting of municipal solid waste. J. Environ. Qual., 25: 776-785.

Boris E. 2004. Caractérisation des substances humiques biomimétiques- Effets sur les végétaux. Thèse de Doctorat, INP Toulouse, France.

Calvet R. 2003a. Le Sol. Propriétés et Fonctions (Tome1). Eds France Agricole : Paris; 456 p.
Calvet R. 2003b. Le Sol. Propriétés et Fonctions (tome 2). Eds France Agricole : Paris; 511 p.

De Nobili M, Bragato G, Alcaniz JM, Puigbo A, Comellas L. 1990. Characterization of electrophoretic fractions of humic substances with different electrofocusing behavior. Soil Science, 150: 763-770.

Domeizel M, Khalil A, Prudent P. 2004. UV spectroscopy: a tool for monitoring humification and for proposing an index of the maturity of compost. Bioresource Technology, 94: 177-184.

Lichtfouse E, Chenu C, Baudin F, Leblond C, Da Silva M, Behar F, Derenne S, Largeau C, Wehrung P, Albrecht P. 1998. A novel pathway of soil organicmatter formation by selective preservation of resistant straight-chain biopolymers: chemical and isotope evidence. Org. Geochem., 28(4): 411-415.

Miano TM, Piccolo A, Celano G, Senesi N. 1992. Infrared and fluorescence spectroscopy of glyphosate-humic acid complexes. The Science of the Total Environment, 123: 83-92.

Niemeyer J, Chen Y, Bollag J-M. 1992. Characterization of humic acids, composts and peat by diffuse reflectance Fourier-Transform infrared spectroscopy. Soil Sci. Soc. Am. J., 56: 135-140.

Peña-Médez EM, Havel J, Patocka J. 2005. Humic substances compounds of still unknown structure: applications in agriculture, industry, environment and biomedicine. J. Appl. Biomed., 3: 13-24.

Schulten H-R, Schnitzer M. 1992. Structural studies on soil humic acids by CuriePoint pyrolysis-gas chromatography/mass spectrometry. Soil Science, 153(3): 29-30.

Schulten, H-R, Schnitzer MA. 1993. State of the art structural concept for humic substances. Naturwissenschaften, 80: 2930 . 
Swift RS. 1996. Organic matter characterization. In Methods of Soil Analysis (Part 3). Chemical Methods, Sparks DL, Bartels JM, Bigham JM (eds). Soil Sci. Soc. Am. Book Series 5: Madison; 1011-1069.

Thomsen M, Lassen P, Dobel S, Hansen PE, Carlsen L, Mogensen BB. 2002. Characterisation of humic materials of different origin: A multivariate approach for quantifying the latent properties of dissolved organic matter. Chemosphere, 49: 327-1337.

Wolf M, Buckau G, Gekeis H, Thang NM, Hoque E, Szynczak W, Kim JI. 2001.
Aspect of measurement of the hydronamic size and molecular mass distribution of humic and fulvic acids. In Humic Substances: Structures, Models and Functions, Ghabour EA, Davies G (eds). Royal Society of Chemistry: UK.; 51-61.

Zbytniewski R, Buszewski B. 2005. Characterization of natural organic matter (NOM) derived from sewage sludge compost. Part 1: chemical and spectroscopic properties. Bioresource Technology, 96: 471-478. 